\title{
Research on practical teaching system of the Internet of things technologies and application
}

\author{
SUN Haiyan \\ School of Ecological Technology and Engineering \\ Shanghai Institute of Technology \\ Shanghai, China \\ e-mail: sj-shy@163.com
}

\begin{abstract}
The Internet of things technologies and application of practice teaching the connotation and features of factors of capability is analyzed in the paper. The Internet of things technologies and application of practice teaching system are put forwarded. And the formation of the system requirements, practice teaching system and content and so on research and discussed in detail.
\end{abstract}

Keywords- Internet of things technoloies; application; practical teaching system; research

\section{INTRODUCTION}

With the implementation of the national strategic development plan, the existing more than five hundred undergraduate institutions of higher learning courses in the Internet of Things technologies and application, is expected to open further expansion in the future. Shanghai Institute of Technology is the first to open the Internet of Things technologies and application course of one of the colleges and universities, and began to set up courses for the master's stage in 2010. This study based on Internet of Things technologies and application of teaching practice, combined with the concept of foreign technical teaching practice mode, discusses the Internet of Things technologies and application of practice teaching system and the implementation ${ }^{11-5]}$.

\section{CURRICULUM ABILITY TRAINING REQUIREMENTS}

Internet of Things technologies and application ability should include information on the overall engineering calculation of thinking ability, arithmetic design and analysis ability, program design and implementation ability, system ability, etc., and embodies the professional ability to Internet of Things perception layer, transport layer, data processing layer, application layer, and complete the Internet of things application system analysis, design and implementation.

Through learning of this course, let the student technology breakthrough point on the Internet of Things Technologies, from the conception, design, implementation and operation of the project is all-round aspects such as the Internet of things technology is applied to the engineering practice, implement close cooperation process of school, society and industry in engineering education.

\author{
LI Xiaobin \\ School of Electrical and Electronic Engineering \\ Shanghai Institute of Technology \\ Shanghai, China \\ e-mail: lixiaobinauto@163.com
}

\section{CONSTRUCT THE PRACTICE TEACHING SYSTEM}

Internet of things and application belong to engineering technology courses. The practical teaching system design should base on of cultivating students' engineering ability and accomplishment. Especially, the ability should to solve the problem of the Internet of things engineering. The Internet of things technology and the application of practice teaching goal is service in the course of study, to deepening understands of Internet course theory knowledge. Preliminary application of curriculum knowledge is cultivated the ability of engineering practice.

Internet of Things technologies and application of practice teaching are closely related to the Internet of things industry, to practical engineering project as the background, engineering technology as the main line, to project implementation as a main line throughout the course of practice teaching. Planning and integration of various kinds of course experiment, designed around the project to organize the experimental teaching system, including course experiment, course design, science and technology innovation activities, production practice, graduation design, etc.

On the basis of Internet of Things technologies and application ability training requirements, build the Internet of Things technologies and application of practical teaching system is shown in Figure.1.

Build the Internet of Things technologies and application of practical teaching center as shown in Figure.2, including the basic experiment platform, professional experiment platform and joint center for cooperating with enterprises. Through the "085 project" construction of Shanghai and the discipline of teachers practical scientific research and engineering projects, successively established for flexible manufacturing electronic products manufactured in the Internet of Things sensor platform, the Internet of Things technologies and application of environmental perception and control center, wireless electronic nose perception laboratory, etc.

And the enterprise to build the Agilent technologies laboratory, Rockwell Automation engineering research center, KUKA robot applications research and development center, Honeywell domestic intelligence center, Internet of Things simulation teaching center, etc. 


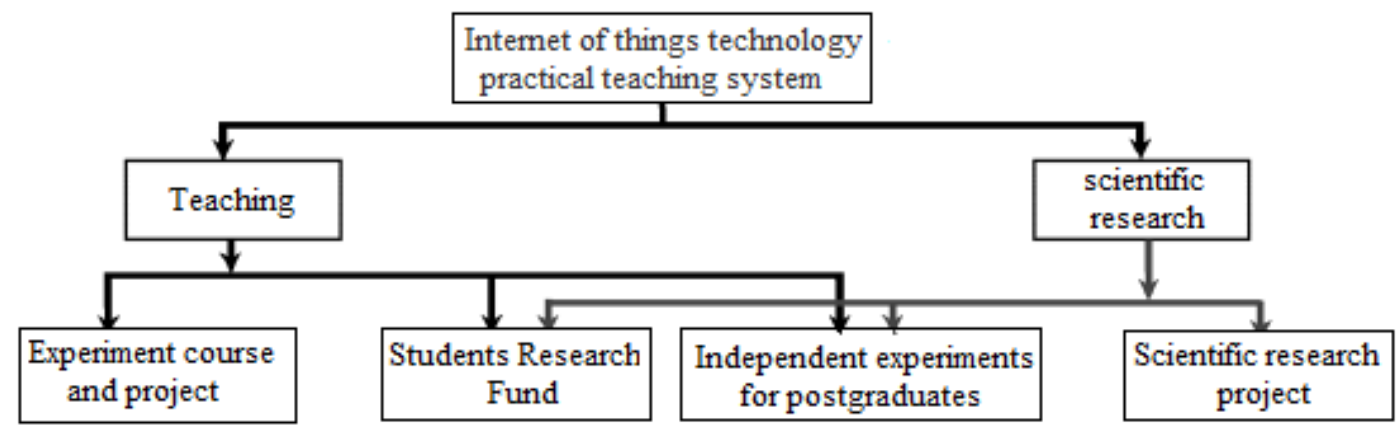

Figure.1 Internet of things technology and application course practice teaching system

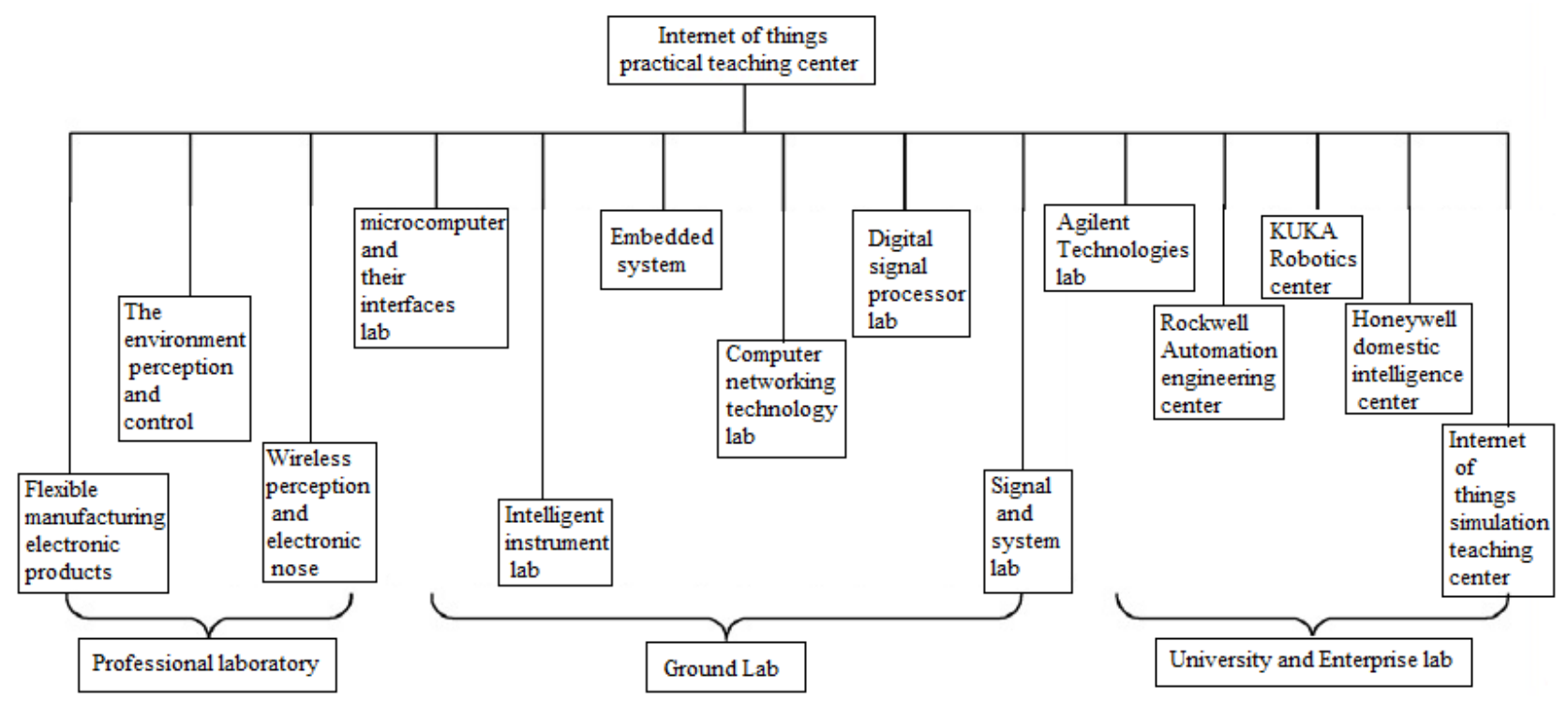

Figure.2 Internet of things technology and the application practical teaching center

\section{DESIGN AND PRACTICE OF TEACHING CONTENT}

The Internet of Things technologies and application of the experimental teaching of the course content gives prominence to the comprehensive ability to use and develop knowledge engineering system analysis and design ability, application direction around the Internet of things technology, set up the establishment of the sensors and sensor networks of flexible manufacturing, electronic products manufacturing, environmental perception and control, electronic nose Laboratory, and the engineering application of the experimental project.

In the Internet of Things application technology teaching experiment, mainly through the experiment center equipment and experiment a variety of radio frequency identification and network hardware such as sensor nodes and routers, as well as the network protocol and the data collection and control software technology, etc. At the same time can be on the ARM processor is the kernel of the gateway, to complete a variety of wireless network management, sensors and radio frequency identification information processing, through different paths, wired and wireless network to transmit data to the center of the Internet of things the server database and internet; And the use of network monitoring software, network monitoring, network maintenance and database management in the computer.

In the experimental system, the experiment is divided into two parts. to do experiment and chosen to do experiments, teaching at different levels. The first level is basic verification experiments. The second level is the comprehensive experiment. The third level is the design experiment. The third level is the study of innovative experiment. This experimental teaching system of multilevel categories, on the one hand can guarantee access to the system of the students with the basic practical ability, to achieve the basic professional requirement; For the student of spare capacity, on the other hand provides innovative practice platform, to achieve the goal of their aptitude.

Internet of things teaching center can not only serve the undergraduate or graduate practice teaching, But also cultivate undergraduate engineering practice ability and 
graduate student's scientific research ability. Through student innovation fund projects and participating in the teachers' scientific research, outstanding undergraduate or graduate student can further carry out research and development In the center of innovation laboratory.Innovation laboratory is given priority to management mode ,with student-based management, teacher- supplemented management as the auxiliary management mode; Every day students are responsible for the daily management in the laboratory on duty, the way is not only cultivate students' innovation ability, but also exercise their ability to lab maintenance and management.

\section{CONCLUSION}

The application of the Internet of things technology and industry has been involved in multiple disciplines. Through the practice teaching system to make the students understand to the interdisciplinary knowledge. In the practice teaching system includes the construction of knowledge system and basic engineering ability training. Through a variety of practice to cultivate the students' engineering quality, practice ability and innovation consciousness. To train high quality applied talents, top creative talents lay a solid foundation. The practical teaching system can also be for computer, electronic information and other related professional students to provide a more professional network experiment platform.

\section{ACKNOWLEDGMENT}

This work was partially supported by Shanghai Institute of Technology graduate students course construction project (4521ZK130059054).

\section{REFERENCES}

[1] State Council of the People's Republic of China. National medium and long-term science and technology development plan (2006-2020) [Z] .[2006-02-01] http://wenku.baidu.com/view/ ddcecbcea1c7aa00b52 acbb6.html.

[2] Qin Leihua,Shi Ke Gan Zaobin. CDIO-based research and practice of practical course system for the specialty of internet of things[J]. Research in Higher Education of Engineering,2013,5,168-172.

[3] Qian Hongyan, Chen Bing, Yan Xuefeng. Research on the Practice Architecture of the Internet of Things[J]. Computer Education,2011,23,2125.

[4] The People's Republic of China ministry of science and technology. “A new generation of broadband wireless mobile communication network "national science and technology major projects 2011 annual grant application guide[Z]. [2010-05-01]. http://www.most.gov.cn/tztg/201005/ W020100512591012346402.doc.

[5] Cheng Yuandong. Developing Trend of Internet of Things Cultivation of Talents in High Vocational Colleges[J]. Internet of Things Technologies,2011,1(2) :41-43. 